\title{
REVIEw: Frank Markham Brown's Boolean Reasoning: The Logic of Boolean Equations
}

\author{
Kari SAUKKONEN \\ Department of Economics, Turku School of Economics \\ kari.saukkonen@tukkk.fi \\ Received by Greg Restall \\ Published July I2, 2005 \\ http://philosophy.unimelb.edu.au/ajl/2005 \\ (c) 2005 Kari Saukkonen
}

Brown, Frank Markham: Boolean Reasoning: The Logic of Boolean Equations. Second edition, New York: Dover, 2003; i-xii, 29I pp. usD\$16.95.

ISBN: 0486427854

\section{INTRODUCTION}

Author Frank Markham Brown, in his book Boolean Reasoning: The Logic of Boolean Equations, aims at an outline of Boolean logic-its mathematical basis, its theory, and its applications. According to the author, Boolean logic proceeds with zero-normal form equations, that is, equations of the form

$$
f\left(x_{1}, x_{2}, \ldots, x_{n}\right)=0
$$

where $\left(\vec{x}_{n}\right) \in B^{n}$ and $B$ is a Boolean algebra. Only finite Boolean algebras are considered.

The Boolean logic presented is based on the abstract Boolean algebra given by Huntington's [I4] postulates and on a special canonical form for a Boolean function given by Archie Blake [2]. The theory of reasoning is divided into functional and general parts, both of which seek antecedents or consequents of the Boolean expressions given. Applications of Boolean reasoning, given by the author, include medical diagnosis, digital fault-location, modeling the adrenal gland, among other things.

Brown's monograph-textbook is for all philosophers, logicians, and mathematicians interested in 2oth century development of the Boole-Schröder [3, 4, 26. algebra of logic and for various scientists, engineers, and medical experts looking for inference techniques that can be applied to Boolean data. A distinctive feature of the book is that it emphasizes reasoning as a primary object of logic as contrasted to Quinean $[\mathrm{I9}, 2 \mathrm{O}, 2 \mathrm{I}, 22]$ formula-minimization. 
The second, revised edition [6] differs from the first edition [5]. It contains new subsections such as "Solving Boolean equations using maps", "Reduction using resolvents", and "Solving Boolean equations using resolvents". Some material is removed, for example, the chapter dealing with Boolean identification now contains only four theorems instead of six. The number of references is significantly reduced, but there are also new references, two of which are by Rudeanu [23, 25].

\section{GENERAL BOOLEAN REASONING}

To have the zero-normal form equation before us in the first place, the given initial information must be represented as a collection of equations. The equations are then condensed into a single equation of the form $f\left(x_{1}, x_{2}, \ldots, x_{n}\right)$ $=0$. The next step is to generate the "prime implicants" of $f$. The third and the last step involves applying reasoning operations to solve the problem.

Note that a given system of Boolean equations may be reduced to a single equation unlike a system of equations in other branches of algebra. From here on, the interest is primarily in the consequents of this equation, not in the solutions of it since the solutions may be just antecedents. Although George Boole himself was mostly occupied with the (functional) solutions of the equation, general methods of Boolean reasoning, both antecedent and consequent, were developed by the Russian logician and mathematician Platon Sergeevich Poretskii in the late igth century, see [27, pp. 216-247]. Blake [2] devised a practical method for obtaining the consequents before the mid-2oth century, which is the approach Brown follows.

Recall that in traditional logic the hypothetical syllogism is an argument of the form if $\mathrm{P}$ then $\mathrm{Q}$, if $\mathrm{Q}$ then $\mathrm{R}$, so if $\mathrm{P}$ then $\mathrm{R}$. Hypothetical syllogism can be translated into the equation-based notation as follows- $P^{c}=0, Q^{c}=0$, so $\mathrm{PR}^{\mathrm{c}}=0$ - which can readily be proved in Boolean algebra (the symbol "c" denotes complementation).

In one of the main chapters of the book Brown outlines a basic reasoning technique that he calls "syllogistic reasoning". It is a simple, but non-trivial (see the Alfred-example pp. 132-135) algebraic reasoning technique which can be applied to statements that are formulated in natural language.

This "syllogistic reasoning" proceeds roughly as follows. The given information is first written as zero-equations. For example, the hypothetical statement "If Alfred studies, then he receives good grades" is first thought as $\mathrm{P} \rightarrow \mathrm{Q}$ and then written as equation $P Q^{c}=0$. All equations are then reduced to one single zero-equation, consisting of the disjunction of each equation's left-sides on the left and zero on the right, like $\mathrm{PQ}^{\mathrm{c}}+\mathrm{QR}^{\mathrm{c}}+\mathrm{PR}^{\mathrm{c}}=0$. The operation, called consensus, which Brown attributes to Quine [I9], is then applied repeatedly to the formula until the simplified canonical form, that is, the Blake canonical form, is produced. The produced formula represents all consequents that may be inferred from the given initial premises. Finally, the formula is 
broken down to a system of single-equations which are translated back into the original language from which one started.

A distinctive feature of "syllogistic reasoning" is that it determines the consequents. Boolean problems are not usually formulated as statements to be proved or disproved. "Syllogistic reasoning" is also related to the resolutionbased reasoning methods used in predicate logic. This concludes our short tour of "syllogistic reasoning".

Let $\vec{f}_{m}: B^{n} \rightarrow B$ be a given collection of Boolean functions. Obviously, there are some specific relations holding among these functions. Brown outlines and investigates three special types of these functional relations.

Recall that a problem concerning whether or not the members of a given set of terms sum to one is a tautology problem. A close relative to this problem is the problem of finding the minimal subset of the set $\left\{f_{1}, f_{2}, \ldots, f_{m}\right\}$ that sums to one. To this end Brown states and proves the following theorem: Let T be a set of terms and let $\vec{A}_{m}$ be Boolean variables. Then $S$ is a minimal sumto-one subset of $T$ iff the product $A_{1} A_{2} \cdots A_{k}$ ( $k$ is equal to cardinality of $S$ ) is a term of the Blake canonical form of the sum having summands $A_{i} t_{i}\left(\vec{x}_{n}\right)$, where $i=1,2, \ldots, m$ and $t_{i}\left(\vec{x}_{n}\right) \in T$.

Another relation that Brown studies is the dependence of sets of Boolean functions on an arbitrary Boolean algebra, since previous studies on dependence have concentrated on special situations, like propositional or switching functions. In addition, Brown investigates the problem of finding the irredundant formula for a Boolean function $f$ which happens to be defined only on some interval $g \leqslant f \leqslant h$ of Boolean functions $g$ and $h$.

\section{Functional Boolean Reasoning: The antecedents}

George Boole and later igth century logicians solved the zero-normal form equation $f\left(x_{1}, x_{2}, x_{3} \ldots\right)=0$ for certain of its arguments in terms of others thus producing equations of the form $x_{1}=g\left(x_{2}, x_{3} \ldots\right)$ which were functional. Because the end-result is a function, the processes and methods described are labelled as "functional reasoning". Literature on this classic topic includes items of Schröder [26], Couturat [9], Rudeanu [24, 25], and many others.

Let $B$ be a Boolean algebra. A particular solution of $f\left(\vec{x}_{n}\right)=0$ is an element $\left(\vec{a}_{n}\right)$ of $B^{n}$ such that $f\left(\vec{a}_{n}\right)=0$ is an identity. A general solution of $f\left(\vec{x}_{n}\right)=0$ is a representation of the set of its particular solutions. For example, if $n$ is equal to one, this representation can be given by the interval $\{x: f(0) \leqslant x \leqslant$ $\left.f^{\mathcal{c}}(1)\right\} \subset B^{1}$ or by the parametric formula $\left\{f(0)+p f^{\mathcal{c}}(1): p \in B\right\}$ where $p$ is a freely chosen member of $B$.

In this context, Brown outlines general solutions, both interval and parametric, using the known method of "successive elimination of variables", and then gives an outline of the use of Karnaugh maps to solve equations containing only a few variables. 
Some theorems related to solutions of zero-normal form equations were the following. First, Brown states the following two theorems, omitting proofs, which are related to the interval-representation of the solutions: (I) A sequence of Boolean functions $\left(\varphi_{0}, \varphi_{1}, \ldots, \varphi_{n}\right)$ which forms a so-called recurrent cover of a given $n$-variable Boolean function $f$ may be constructed directly from the prime implicants of $f$. (2) Each n-variable Boolean system, called a "subsumptive general solution" of the $n$-variable zero-normal form equation $f$, is associated with a recurrent cover $\left(\varphi_{0}, \varphi_{1}, \ldots, \varphi_{n}\right)$ of the function $f$.

In addition, Brown states and proves an old theorem of Löwenheim I8] which is related to the parametric general solutions. Löwenheim's theorem can be used to form a parametric general solution in a mechanical way from any given particular solution.

Thirdly, Brown states results that are related to the solutions of one-normal form equation $g\left(\vec{x}_{n}\right)=1$, where $n$ is supposed to be a relatively small number. The author discusses first how to present the one-normal form equation as a special map called a Karnaugh map. The g-map may then be used to find all particular solutions of $\mathrm{g}\left(\vec{x}_{\mathfrak{n}}\right)=1$.

A Boolean function $h:\{0,1\}^{n} \rightarrow\{0,1\}$ and its corresponding Karnaugh map is said to be orthonormal if $h$ multiplied with itself with distinct arguments is equal to zero, and the sum of all values is equal to one. Given a Boolean system $S\left(\vec{x}_{n}\right)$ consisting of k-equations each with $n$-variables over a Boolean algebra $B$, its resolvent $\left|S\left(\vec{x}_{n}\right)\right|$, is defined as a product over the system if each equation $g_{i}\left(\vec{x}_{n}\right)=h_{i}\left(\vec{x}_{n}\right)$ is first replaced with the expression $g_{i}\left(\vec{x}_{n}\right) \odot h_{i}\left(\vec{x}_{n}\right)$, where $\odot$ is the equivalence-operation, that is, the exclusive NOR.

Brown states and proves the following theorem which is not included in the first edition of the book. Let $g_{0}=1$ be the consistency-condition of the one-normal form equation $\mathrm{g}\left(\vec{x}_{\mathfrak{n}}\right)=1$, meaning that there is at least one solution. Then the $n$-variable function $h$ is equal to the resolvent $\left|\left(\vec{x}_{n}\right)=\varphi\right|$ of a particular solution $x_{1}=\varphi_{1} \in \mathrm{B}, x_{2}=\varphi_{2} \in \mathrm{B}, \ldots, x_{n}=\varphi_{n} \in \mathrm{B}$ of the equation $g\left(\vec{x}_{n}\right)=1$, iff $h$ is orthonormal and $h$ is included in the sum $g\left(\vec{x}_{n}\right)+g_{0}^{c}$.

\section{Functional Boolean Reasoning: the Consequents}

Producing the functional antecedents of the zero-normal form or the onenormal form equation is only one part of the "functional reasoning". The other part of "functional reasoning" consists of finding functional consequents of this type of equation. If $f\left(\vec{x}_{n}\right)=1$ is a consistent one-normal form equation, the task is to find consequents of this equation having the form $x_{1}=$ $g\left(x_{2}, x_{3}, \ldots, x_{n}\right)$. This "functional deduction", as Brown titles it, seems to have received little attention in the literature. Brown refers to Ledley's [I6], [I7] work in this context.

Consider a Boolean system $S$ consisting of equations $u_{i}=g_{i}\left(\vec{v}_{s}\right)$ indexed 
by $i=1,2, \ldots, r$, such that $\left(\vec{u}_{r}\right)$ and $\left(\vec{v}_{s}\right)$ are disjoint subvectors of $\left(\vec{x}_{n}\right)$ and $\vec{g}_{r}: B^{S} \rightarrow B$ are s-variable Boolean functions. A Boolean system $S$ is said to be a functional consequent of $f\left(\vec{x}_{n}\right)=1$, and each argument $\left(\vec{u}_{r}\right)$ is said to be functionally deducible, in case $f\left(\vec{x}_{n}\right)=1$ implies $S$.

Brown studies only one-equation systems, that is, systems where $r=1$. The main problem is to determine the minimal sets of variables from which the value of variable $u$ may be computed, given that $u$ is functionally deducible from $f\left(\vec{x}_{n}\right)=1$.

Let $\{\{u\}, V, W\}$ be a collection of subsets of $\left\{x_{1}, x_{2}, \ldots, x_{n}\right\}$ having the property that each argument $x_{i}$ of the equation appears in exactly one of these subsets. The set $W$ is said to be $u$-eliminable from $f\left(\vec{x}_{n}\right)=1$ if $f\left(\vec{x}_{n}\right)=1$ implies $u=g(V)$.

Let $f: B^{n} \rightarrow B$ be a Boolean function expressed in terms of arguments $\vec{x}_{n}$,

and let $R, Q$, and $T$ be subsets of $\left\{x_{1}, x_{2}, \ldots, x_{n}\right\}$. Define another Boolean function called the "disjunctive eliminant" of $f$ with respect to the subset $T$, denoted by $\operatorname{EDIS}(f, T)$, as follows: (I) $\operatorname{EDIS}(f, \emptyset)=f,(2) \operatorname{EDIS}\left(f,\left\{x_{1}\right\}\right)=f\left(0, x_{2}, \ldots, x_{n}\right)+$ $f\left(1, x_{2}, \ldots, x_{n}\right),(3) \operatorname{EDIS}(f, R \cup Q)=\operatorname{EDIS}(\operatorname{EDIS}(f, R), Q)$.

Brown states and proves the following theorem: $A$ subset $W$ is $u$-eliminable from the one-normal form equation $f\left(\vec{x}_{\mathfrak{n}}\right)=1$, if and only if $u$ is functionally deducible from the equation in which the disjunctive eliminant with respect to subset $W$ is equal to 1 . Brown then outlines a method which generates the minimal $u$-determining subsets.

\section{CHOICE OF THE CANONICAL FORM}

Next, a few remarks about the choice of the canonical form is made. A Boolean function $f$ determines a certain formula such that another formula (if congruent to it) may be produced using only the commutative law. Blake called this formula the simplified canonical form, Brown calls it the Blake canonical form.

There are several different ways of representing a Boolean function. One example is the minterm form, discussed also in Boole [4, 66-79]. For example, if $f: B^{2} \rightarrow B$ is a Boolean function, then by Boole's expansion theorem [4, pp. 66-79] $f\left(x_{1}, x_{2}\right)=x_{1}^{c} f\left(0, x_{2}\right)+x_{1} f\left(1, x_{2}\right)$ for all $\left(x_{1}, x_{2}\right) \in B^{2}$. Repeated application of Boole's expansion theorem shows that

(*) $\quad f\left(x_{1}, x_{2}\right)=x_{1}^{c} f\left(0, x_{2}\right)+x_{1} f\left(1, x_{2}\right)=x_{1}^{c} x_{2}^{c} f(0,0)+x_{1}^{c} x_{2} f(0,1)$ $+x_{1} x_{2}^{c} f(1,0)+x_{1} x_{2} f(1,1)$.

The values $f(0,0), f(0,1), f(1,0), f(1,1)$ are elements of the carrier of $B$, that is, of the underlying set of the algebra $B$, and they are called the discriminants of the function $f$. The discriminants of $f$ carry all of the information concerning the nature of $f$. The expanded equation $(*)$ is called the minterm canonical form of $f$.

Since there is only one minterm realization of any Boolean function, why 
not use the minterm form? John Gregg [12, p. 177] notes that some Boolean mathematicians use minterm form as their canonical form and have no problem with it. But he [I2, p. I77] adds that the minterm expressions often can be more complicated than one would think that they should be.

According to Gregg [12, pp. I8I-I82] the Blake canonical form is a kind of simplification of a given lengthy expression, but it is the fact that the form contains all of the prime implicants of the function that makes it at least as appealing as the simplifying properties. It enables one to "ferret out hidden implicants and, therefore, hidden logical conclusions from a given set of premises." Gregg [I2, p. I82] even goes to say: "The power of this method, when applied to the field of logic, is something even Aristotle could not have imagined."

Brown's tacit argument to base Boolean reasoning on the Blake canonical form, and not for some other form or representation, is its alleged practicality in reasoning. For example, Poretskıı's Boolean reasoning system, see [27, pp. 216-247], was based on tables of antecedents and consequents, but according to Brown, they are of little practical use for the tables grow rapidly with the number of variables used. According to Brown, Blakes's method is more practical than Poretskii's.

\section{THE METHOD OF REASONING AND PROOF}

This last section discuss briefly the method of reasoning and proof. Logicians involved with lattices, topologies, measures, sets and the like, may not bother to ask justifications for developing the reasoning methods based on Boolean algebra; on the other hand logicians who have philosophical training may wonder what to do about Boole since it is known that Gottlob Frege [II] opened the path to modern reasoning anyway and that reasoning is to proceed with quantifiers and predicates, not by Boolean equations. But to think like a postBoolean differs fundamentally from thinking like a post-Fregean or traditional logician.

Note that a Boolean function $f: B^{n} \rightarrow B$ takes values over the carrier of its algebra. The image-set of this mapping being, as shown by Schröder [26, vol. I, sct. 19], the interval $[\Pi f(A), \Sigma f(A)]$, where $A \in\{0,1\}^{n}$. In the two-valued Boolean algebra, which has the carrier $\{0,1\}$, the following properties hold:

$$
\begin{aligned}
& x+y=1 \text { implies } x=1 \text { or } y=1 \\
& x y=0 \text { implies } x=0 \text { or } y=0 \\
& x \neq 1 \text { implies } x=0 .
\end{aligned}
$$

These properties hold in classical logic, but they do not hold in Boolean algebras whose carriers consists of more than two elements. According to Brown, these "big" Boolean algebras can not be avoided, at least in the context of switching systems.

In Aristotle's (384-322 BC) syllogistic logic [II, and in the classical proposi- 
tional and predicate logic since, deductions are considered to have two basic forms: direct and indirect. The rule of inference that permits inferring $P$ having derived a contradiction from not- $\mathrm{P}$ is an example of an indirect inference rule. This rule, often called reductio ad absurdum, is the basic principle in classical logic and in every-day mathematics; however, this inference rule is not possible in Boolean logic since, according to Brown, the denial of a Boolean equation is not a Boolean equation. John Corcoran [7, p. 28o], [8, pp. xxvixxix] says that Boolean reasoning, or at least Boole's [4] original work, is flawed since it does not permit this indirect form of inference.

Of course, this version of reductio ad absurdum to which Corcoran [7, p. 280$]$, [8, p. xxvi] is referring to does not hold in many other logics either, for example, it does not hold in intuitionistic logic. The rule is also related to the notion of complementation in the algebra used. According to Dunn and Hardegree [IO p. 90] the classical principles of negation can be formulated lattice-theoretically as follows:

$$
\begin{aligned}
& \text { (PI) } x \leqslant x^{c c} \\
& \text { (P2) } x \leqslant y \text { implies } y^{c} \leqslant x^{c} \\
& \text { (P3) } x^{c c}=x \\
& \text { (P4) } x \wedge x^{c}=0 \\
& \text { (P5) } x \vee x^{c}=1
\end{aligned}
$$

Although Heyting's [13] intuitionistic logic rejects principles $\mathrm{P}_{3}$ and $\mathrm{P}_{5}$, the following version of the reductio ad absurdum rule still holds in the intuitionistic logic: Infer not-P having derived a contradiction from P. So intuitionistic logic permits a form of indirect reasoning. It is also interesting to note that Heyting algebra, see [15, pp. I-38] or [25, pp. 38-6o], is a more general notion than Boolean algebra. Principles like $\mathrm{P} 3$ and $\mathrm{P} 5$ are readily seen to hold in the Huntington-type abstract Boolean algebra. In fact, it can be shown that a Heyting algebra is a Boolean algebra if the principle $\mathrm{P} 3$ holds for every element of the algebra.

This does not remove the fact that one can not take negation of a Boolean equation and expect the result to be a Boolean equation: as Brown remarks, denying the equation $a=b$ is not the same thing as, for example, asserting the equation $a=b^{c}$. However, according to Brown, in most applications, a Boolean problem is not formulated as a theorem to be proved, the task is rather to determine the consequents. This involves "forward chaining" to produce the Blake canonical form, and the "forward chaining" can serve no purpose in predicate logic.

\section{REFERENCES}

[r] Aristotle. Prior Analytics. Indianapolis: Hackett, I989. 
[2] Blake, Archie: Canonical Expressions in Boolean Algebra. University of Chicago, 1938.

[3] Boole, George: The Mathematical Analysis of Logic, Being an Essay towards a Calculus of Deductive Systems. Oxford: Basil Blackwell, I95I, [1847].

[4] Boole, George: The Lawes of Thought. New York: Prometheus, 2003, [1854].

[5] Brown, Frank Markham: Boolean Reasoning. The Logic of Boolean Equations. Boston, Kluwer 1990.

[6] Brown, Frank Markham: Boolean Reasoning. The Logic of Boolean Equations. New York, Dover 2003. 2nd ed.

[7] Corcoran, John: "Introduction". In Boole [4, pp. vii-xxxv].

[8] Corcoran, John: "Aristotle's Prior Analytics and Boole's Lawes of Thought". History and Philosophy of Logic, 24, 2003, 26I-288.

[9] Couturat, Louis: The Algebra of Logic. Chicago: Open Court, I9I4, [1905].

[ro] Dunn, Michael, J.-Hardegree, Gary, M.: Algebraic Methods in Philosophical Logic. Oxford: Oxford University Press, 200I.

[II] Frege, Gottlob: Begriffsschrift. A Formula Language, Modeled upon that of Arithmetic, for Pure Thought. In Heijenoort, Jean van (Ed.): Frege and Gödel. Tro Fundamental Texts in Mathematical Logic. New York: Harvard University Press, 1999, [1879].

[12] Gregg, John: Ones and Zeros. Understanding Boolean Algebra, Digital Circuits, and the Logic of Sets. New York: IEEE Press, 1998.

[13] Heyting, A.: "Die formalen Regeln der intuitionistischen Logik". In Berka, Karel-Kreiser, Lothar (eds.): Logik-Texte. Kommentierte Auswahl zur Geschichte der Modernen Logik. Berlin: Akademie-Verlag, 1971, I73-178, [1930].

[14] Huntington, Edward V.: "Sets of Independent Postulates for the Algebra of Logic". Transactions of the American Mathematical Society, 5, 1904, 288304 .

[15] Johnstone, Peter, T:: Stone Spaces. Cambridge: Cambridge University Press, 1982.

[16] Ledley, Robert S.: "Digital Computational Methods in Symbolic Logic, with examples in biochemistry". Proceedings of the National Academy of Sciences of the United States of America, 4I, I955, 498-5II.

[17] Ledley, Robert S.: Digital Computer and Control Engineering. New York: McGraw-Hill, r960. 
[18] Löwenheim, Leopold: "Über die Auflösung von Gleichungen im logischen Gebietekalkul”. Mathematische Annalen, 68, I9I0, I69-20I.

[19] Quine, W. V.: "The Problem of Simplifying Truth Functions". The American Mathematical Montbly, 59, 1952, 52I-531.

[20] Quine, W. V.: "Two Theorems about Truth Functions". Boletín de la Sociedad Mathemática Mexicana, ı, 1953, 64-70.

[2I] Quine, W. V:: "A Way to Simplify Truth Functions". The American Mathematical Monthly, 62, 1955, 627-631.

[22] Quine, W. V.: “On Cores and Prime Implicants of Truth Functions”. The American Mathematical Monthly, 66, 1959, 755-760.

[23] Rudeanu, Sergiu: "Solutions non redondantes des équations booléennes". Bulletin Mathématique de la Société des Sciences Mathématiques et Physiques de la République Populaire Roumaine, 7, 1963, 45-49.

[24] Rudeanu, Sergiu: Boolean Functions and Equations. Amsterdam: NorthHolland \& Elsevier, I974.

[25] Rudeanu, Sergiu: Lattice Functions and Equations. London: Springer, $200 \mathrm{I}$.

[26] Schröder, Ernst: Vorlesungen über die Algebra der Logik. Leipzig: Teubner, I890-I895.

[27] Styazhkin, N. I.: History of Mathematical Logic from Leibniz to Peano. Cambridge: The M.I.T. Press, I969, [1964]. 